\title{
BMJ Open Development of a population pharmacokinetic model of olanzapine for Chinese health volunteers and patients with schizophrenia
}

\author{
Anning Li, ${ }^{1}$ Shuangmin $\mathrm{Ji}^{2}$ Weihua Yue, ${ }^{3}$ Hao Yan, ${ }^{3}$ Fang Dong, ${ }^{1}$ Canjun Ruan, ${ }^{1}$ \\ Wenbiao Li, ${ }^{1}$ Wei Lu, ${ }^{4}$ Dai Zhang, ${ }^{3,5}$ Chuanyue Wang ${ }^{1}$
}

To cite: Li A, Ji S, Yue W, et al. Development of a population pharmacokinetic model of olanzapine for Chinese health volunteers and patients with schizophrenia. BMJ Open 2018;8:e020070. doi:10.1136/ bmjopen-2017-020070

- Prepublication history for this paper is available online. To view these files, please visit the journal online (http://dx.doi org/10.1136/bmjopen-2017020070).

$\mathrm{AL}$ and $\mathrm{SJ}$ contributed equally. DZ and CW contributed equally.

Received 13 0ctober 2017

Revised 21 March 2018

Accepted 3 April 2018

Check for updates

(c) Author(s) (or their employer(s)) 2018. Re-use permitted under CC BY-NC. No commercial re-use. See rights and permissions. Published by BMJ.

For numbered affiliations see end of article.

Correspondence to Professor Chuanyue Wang; wang_cy@ccmu.edu.cn

\section{ABSTRACT}

Objective Olanzapine is an atypical antipsychotic drug commonly used for the treatment of schizophrenia. However, there are still many complications associated with the use of olanzapine, and researchers continually strive to improve the handling of data from regular therapeutic drug monitoring (TDM). The objective of this study is to optimise the individualised treatment of olanzapine by establishing a population pharmacokinetics (PopPK) model in Chinese patients with schizophrenia. Methods This study integrates an extensive collection of concentration data from healthy volunteers after a single dose and a less extensive collection of samples from patients undergoing TDM. A PopPK model was developed using non-linear mixed-effects modelling. Potential covariates, including the olanzapine manufacturer and patient gender and age, were assessed during model development. A total of 616 plasma concentration levels from 22 healthy male individuals in China and 458 concentration levels from 112 male and 122 female patients with schizophrenia undergoing TDM at 12 hospitals in China were included in the analysis. The concentration profile could be best described using a two-compartment model with first-order absorption and elimination.

Results The absorption rate (Ka) of olanzapine ranged from $2.85 h^{-1}$ to $5.39 h^{-1}$ for the different formulations. The typical absorption time delay was 0.877 hour. Body weight had a considerable effect on the apparent volume of the centre compartment and showed a power relationship. Conclusions A PopPK model of olanzapine in Chinese patients with schizophrenia was developed in this study. After determining the PK parameters of olanzapine, the results suggested that body weight exhibited a considerable impact effect on $V_{c} / F$. The impact of subjects and formulations requires further study. The PopPK model established in this study is likely to provide some information for the individualised therapy of olanzapine. Trial registration number ChiCTR-TRC-10000934; Results.

\section{INTRODUCTION}

Schizophrenia is among the most severe mental disorders with $1 \%$ of the population having a chance of developing schizophrenia
Strengths and limitations of this study

- The data were used for the development of a population pharmacokinetics (PopPK) model for Chinese in this study, including both healthy volunteers and patients with rich data and sparse data.

- All data applied in this manuscript were retrospectively collected from completed studies.

- A two-compartment model with first-order absorption and elimination describes the PopPK characteristics of olanzapine in a Chinese population very well.

- The impact of some covariates, subjects and formulations could not be thoroughly identified in this study.

- PopPK parameters obtained from this study would likely help to individualise the use of olanzapine for Chinese patients, which has not been well investigated.

during their lifetime. ${ }^{1}$ It is most commonly observed in young adults and comprises multiple characteristic disorders involving thinking, perception, emotion and behaviour. The course of the disease is often protracted, and more than half of the diagnosed patients gradually lose their social functioning abilities.

Olanzapine is an atypical antipsychotic drug commonly used in China. Its rate of prescription $(13.1 \%)$ makes it the second most prescribed of all atypical and antipsychotic drugs in China. In addition, it has had the greatest increase in prescription rate among all atypical antipsychotic drugs, revealing its considerable usage. ${ }^{2}$ In a study performed in China investigating 26 randomised controlled trials, a positive and negative syndrome scale assessment found a mean reduction value of 44.3 points with olanzapine treatment, suggesting that the drug has a positive effect for the treatment of schizophrenia. ${ }^{3}$ The National Institutes 
of Mental Health-funded Clinical Antipsychotic Trials of Intervention Effectiveness study in the USA indicated that $74 \%$ of 1493 subjects terminated treatment with olanzapine within 18 months. The dropout rate among subjects who were first assigned to the olanzapine group was $68 \%$, which was lower than the dropout rate for users of other atypical antipsychotic drugs, such as risperidone, quetiapine and ziprasidone. ${ }^{4}$

This high dropout rate is most likely associated with insufficient treatment efficacy and intolerance of the side effects. The metabolism of olanzapine in humans shows wide and significant interindividual variability (IIV). A previous study revealed that the difference in the clearance rate of olanzapine among individuals was 4-fold to 10 -fold, resulting in a large variance in drug concentration after administration of the same dose of olanzapine. ${ }^{56}$ Some patients may not receive an effective dose, resulting in poor control, while other patients may experience obvious side effects that can lead them to terminate the use of the drug.

Therapeutic drug monitoring (TDM) is an important method for ensuring drug efficacy and controlling side effects in the clinical practice. The data collected from TDM are quite valuable because they include a variety of patients and reflect real clinical responses to olanzapine. It is important to better use these data to provide more information for individualised therapy. A population pharmacokinetics (PopPK) model is a method for simultaneously analysing both rich and sparse data to identify the sources of variation in pharmacokinetic (PK) parameters.

After a PopPK model of olanzapine has been established, individual parameters can be predicted for patients based on the TDM concentration via the Bayesian feedback method. ${ }^{7}$ Few studies have been conducted which examine the PopPK of olanzapine. ${ }^{58}$ The aim of this study is to establish a PopPK model of olanzapine for the Chinese population, and furthermore, to explore the factors that influence the relevant PK parameters.

\section{MATERIALS AND METHODS}

\section{Subject data and study design}

This research included two parts (cohorts A and B), and all subjects were Han Chinese. The subjects from cohort A were added over a 5-month period from February to June 2001 at the Beijing Anding Hospital phase I clinical trial unit. This cohort received olanzapine for 2 months, and concentration measurements were taken during the final 2 months of the study period. All the participants in cohort A were healthy male volunteers who received a $10 \mathrm{mg}$ single dose of olanzapine 'formulation \#0' or 'formulation \#1'. Blood samples were collected 1, 2, 3, 4, $6,8,12,24,36,48,72,96,120$ and 168 hours after drug administration to determine the drug concentrations in the blood at each time point. After a 3-week washout period, the subjects were switched to another drug type, and the same sampling design was repeated.
The subjects from cohort B had sparse available data and were included from May 2010 until December 2011. All patients with schizophrenia were in an open multicentre study. ${ }^{9}$ This study was conducted at five main centres, including 25 hospitals. While a total of 4773 patients were enrolled, our study only included those taking olanzapine. Blood samples were taken for concentration determination at the time of enrolment. These subjects received a DSM-IV-TR-structured clinical interview diagnosis and were diagnosed with schizophrenia. The subjects in cohort $\mathrm{B}$ received olanzapine treatment for 6 weeks. Blood samples were collected at the end of the fourth and sixth week after the initial dose. The majority of blood sample collections were performed at 06:00 hours, although some samples were collected at 22:00 hours. The time of collection was recorded in detail. In cohort B, the dosage of olanzapine was adjusted over 2 weeks, and steady state was achieved. In addition, the subjects were not allowed to take liver enzyme inducers or inhibitors (such as rifampin, warfarin, carbamazepine, phenobarbital, phenytoin and St. John's Wort) for 2 weeks prior to enrolling in the study and throughout the study period.

Olanzapine from three manufacturers was used in this study. The olanzapine used in cohort A was labelled 'formulation \#0' or 'formulation \#1', while the olanzapine used in cohort B was labelled 'formulation \#2'.

Demographic data along with dose-regimen information from subjects of both cohorts have been collected. All the subjects provided written informed consent before joining the study. The registry number is ChiCTR-TRC-10000934. The authors do not have access to information that could identify individual participants during or after data collection.

\section{Sample measurement methods}

Plasma concentrations of olanzapine were analysed by using a validated HPLC-MS/MS method. The assay range was from 2 to $400 \mathrm{ng} / \mathrm{mL}$. Precision and accuracy were evaluated by analysing quality control (QC) samples at $2 \mathrm{ng} / \mathrm{mL}, 4 \mathrm{ng} / \mathrm{mL}, 160 \mathrm{ng} / \mathrm{mL}$ and $320 \mathrm{ng} / \mathrm{mL}$. The interday relative SDs and intraday precision were both within $15 \%$, and the accuracy was within the range of $85 \%-115 \%$. The extraction recoveries of the QC samples were above $90 \%$ for olanzapine.

\section{Model development}

This study used non-linear mixed-effect modelling (NONMEM) to establish a PopPK model for olanzapine. The model was performed using NONMEM (V.7, level 2, ICON Development Solutions, Ellicott City, Maryland, USA) and R (V.3.0.1) ${ }^{10-12}$ Various absorption and elimination models were tested: (1) first-order absorption with a one-compartment model, (2) first-order absorption with a two-compartment model and (3) first-order absorption with time delay and a two-compartment model. First, the intensive data from cohort A were used to establish the basic structure model of olanzapine. Next, the data 
from cohorts A and B were used to establish the final model. Finally, during the model-building process, a firstorder condition estimation with interaction method was applied. The ADVAN4 subroutine was used during the model development process.

Based on current knowledge and experience in PopPK, we assumed that the IIV of PopPK parameters conformed to a log-normal distribution, which could be expressed using the following equation:

$$
P_{i}=P_{p o p} \cdot \operatorname{EXP}\left(\eta_{i}\right)
$$

where $P_{i}$ is the predicted value of the parameter for the individual $\mathrm{i}, \mathrm{P}_{\text {pop }}$ is the population typical value of the parameter, and $\eta_{j}$ is a random variable, identically distributed with a mean of zero and variance of $\omega,{ }^{2}$ that was used to describe the variation for the ith patient.

Residual random effects were considered as random effects within individuals and between experiments as well as unexplained partial differences in random variability between certain individuals. Additive, proportional and mixed error models were all assessed to describe the unexplained error in this model. The error models could be expressed using the following equation:

$$
\begin{array}{r}
\text { Additive error model : Obs }=\text { Pred }+\varepsilon \\
\text { Proportional error model : } \text { Obs }=\text { Pred } \cdot(1+\varepsilon) \\
\text { Mixed error model : Obs }=\text { Pred } \cdot\left(1+\varepsilon_{1}\right)+\varepsilon_{2}
\end{array}
$$

where Obs is the observation, Pred is the predicted value and $\varepsilon$ is the normally distributed random error.

The covariates screened in this study included the following variables: drug manufacturer, age, height, weight, body mass index, gender, aspartate aminotransferase levels, alanine aminotransferase levels as well as blood, urine and nitrogen levels. Linear, exponential and power functions were used to assess these covariates.

The procedure to investigate the covariates included two steps: the first step was forward inclusion to establish the full model; the second step was backward elimination to acquire the final model. In the forward inclusion process, we introduced one covariate into the base model with each repetition, and the degree of freedom was increased by 1 for the model. An objective function value $(\mathrm{OFV})$ reduced to a level of $\mathrm{p}<0.05$ (the OFV decreased by 3.84) indicated that the covariate had a significant effect on the model fitting. The full model was obtained when all covariates with significant effects were introduced into the model. We then eliminated these covariates one by one from the model and determined whether the increase in the OFV could achieve a value of $\mathrm{p}<0.01$ (6.63). If the covariate achieved this significance level, it remained in the model; otherwise, the effect was eliminated.

\section{Model validation}

The bootstrap method was used to assess the stability of the final model. This method repeatedly sampled partial data from the original dataset. For 500 samples in this study, the parameters for each subgroup dataset were calculated. Stability was evaluated based on the frequency distribution of these parameters. ${ }^{13}$

The final model was also validated using normal prediction distribution error (NPDE). NPDE was suitable for the design of multiple-dose drug administration with limited sampling points. A total of 1000 simulations were performed to generate a corresponding NPDE value for the verification of the final model. ${ }^{14}$

\section{Patient and public involvement}

There was no patient or public involvement in this study.

\section{RESULTS}

Twenty-four subjects were initially included in cohort A. Two subjects dropped out due to low compliance, resulting in a total of 22 subjects included in this study. A total of 616 blood samples were collected for cohort A, while cohort B included 458 samples from 234 patients. Among these, 19 samples were taken randomly, and the rest were steady-state concentrations. Detailed information for cohorts A and B is shown in table 1.

A two-compartment model with mixed error best described the PK profile. The final model suggests that the absorption rate constant (Ka) of formulation \#0 differed from that of formulation \#1 and indicates that body weight affects the $V_{C} / F$. The results also show that the manufacturer may affect the apparent central volume of distribution $\left(\mathrm{V}_{\mathrm{C}} / \mathrm{F}\right)$ and apparent elimination rate $(\mathrm{CL} / \mathrm{F})$. This preliminary finding should be validated with more data.

The absorption time delay was 0.877 hour. The typical values for the central compartment volume $\left(\mathrm{V}_{\mathrm{C}} / \mathrm{F}\right)$ and the $\mathrm{CL} / \mathrm{F}$ are shown in table 2.

The final model result suggests that weight had an effect on the $\mathrm{V}_{\mathrm{C}} / \mathrm{F}$ that could be expressed by Equation 5 . Based on the data from this study, the $V_{C} / F$ values of subjects with extreme body weight values ( $36 \mathrm{~kg}$ or $98 \mathrm{~kg}$ ) were as follows for each drug manufacturer: olanzapine formulation \#0 was $389 \mathrm{~L}$ or $694.6 \mathrm{~L}$, respectively; formulation \#1 was $371 \mathrm{~L}$ or $663 \mathrm{~L}$, respectively and formulation \#2 was $1768 \mathrm{~L}$ or $3157 \mathrm{~L}$, respectively. Detailed information for the remaining parameter estimations is listed in table 2. The proportional error was $21.6 \%$, and the additive error was $0.303 \mathrm{ng} / \mathrm{mL}$, which were relatively small. We also calculated the correlation between the CL/F and $\mathrm{V}_{\mathrm{C}} / \mathrm{F}$. The relative $\mathrm{SE}$ of all the parameters was less than $30 \%$, indicating that the parameters were estimated with good precision and gave reliable results.

$$
\begin{aligned}
& \text { For formulation } \# 0: \frac{\mathrm{V}_{\mathrm{C}}}{F}=2390 \times\left(\frac{\mathrm{WT}}{60.59}\right)^{0.579} \times 0.22 \times e^{\eta} \\
& \text { For formulation \#1: } \frac{\mathrm{V}_{\mathrm{C}}}{F}=2390 \times\left(\frac{\mathrm{WT}}{60.59}\right)^{0.579} \times 0.21 \times e^{\eta} \\
& \text { For formulation \#2: } \frac{\mathrm{V}_{\mathrm{C}}}{F}=2390 \times\left(\frac{\mathrm{WT}}{60.59}\right)^{0.579} \times e^{\eta} \\
& \text { For formulation \#0 and \#1: } \frac{C L}{F}=25.4 \times 0.61 \times e^{\eta} \\
& \text { For formulation \#2: } \frac{C L}{F}=25.4 \times e^{\eta}
\end{aligned}
$$

The goodness-of-fit plots for the final proposed models are presented in figure 1. The success rate for the 
Open access

Table 1 Demographic data for the population used for the model in this study

\begin{tabular}{llll} 
Characteristic & Cohort A & Cohort B & Total \\
\hline No. of subjects & 22 & 234 & 256 \\
Drug type & 0,1 & 2 & $0,1,2$ \\
\hline Formulation \#0 & 22 & 0 & 22 \\
\hline Formulation \#1 & 22 & 0 & 22 \\
Formulation \#2 & 0 & 234 & 234 \\
No. of samplings & 616 & 458 & 1074 \\
Male/female & $22 / 0$ & $112 / 122$ & $134 / 122$ \\
\hline Age (year) & $23.0(19.0-39.0)(24.9 \pm 5.7)$ & $30.0(18.0-48.0)(30.9 \pm 7.9)$ & $29.5(18.0-48.0)(30.4 \pm 7.9)$ \\
Body weight (kg) & $62.5(53.5-91.0)(65.3 \pm 9.9)$ & $60.0(36.0-98.0)(60.2 \pm 11.1)$ & $60.0(36.0-98.0)(60.6 \pm 11.1)$ \\
Body height (cm) & $172.0(158.0-180.0)(171.0 \pm 5.8)$ & $163.5(145.0-190.0)(164.2 \pm 8.0)$ & $165.0(145.0-190.0)(164.8 \pm 8.1)$ \\
BMI (kg/m²) & $22.1(17.9-28.1)(22.3 \pm 2.7)$ & $21.9(15.0-36.2)(22.3 \pm 3.5)$ & $21.9(15.0-36.2)(22.3 \pm 3.4)$ \\
AST (U/L) & $20.0(14.0-32.0)(20.5 \pm 4.6)$ & $16.6(1.0-182.0)(23.4 \pm 21.1)$ & $17.0(0.7-182.0)(23.1 \pm 20.2)$ \\
ALT (U/L) & $15.5(6.0-32.0)(16.9 \pm 7.3)$ & $21.0(2.0-147.0)(24.4 \pm 13.8)$ & $20.9(2.0-147.0)(23.8 \pm 13.5)$ \\
BUN (mmol/L) & $4.4(2.3-7.7)(4.5 \pm 1.2)$ & $3.9(1.1-9.8)(4.2 \pm 1.5)$ & $3.9(1.1-9.8)(4.2 \pm 1.5)$ \\
\hline
\end{tabular}

Data are expressed as median (range) (mean $\pm \mathrm{SD})$.

ALT, alanine aminotransferase; AST, aspartate aminotransferase; BMI, body mass index; BUN, blood urea nitrogen.

sampling of 500 bootstraps was $98.8 \%$. The estimated values for the majority of the parameters were similar to the average values obtained by the bootstrap method, except for the parameters for Ka and for the correlation between $\mathrm{CL} / \mathrm{F}$ and $\mathrm{Vc} / \mathrm{F}$. In addition, the $95 \% \mathrm{CI}$ had a narrow range and contained the typical estimates from

Table 2 Parameter estimates of the model and parameter results from a non-parametric bootstrap

\begin{tabular}{|c|c|c|c|c|}
\hline \multirow[b]{2}{*}{ Description } & \multirow[b]{2}{*}{ Estimate } & \multirow[b]{2}{*}{ RSE* $^{*}$} & \multicolumn{2}{|l|}{ Bootstrap } \\
\hline & & & Mean & $95 \% \mathrm{Cl}$ \\
\hline $\mathrm{Ka}\left(\mathrm{h}^{-1}\right)$ & 2.85 & $8.7 \%$ & 2.24 & 1.21 to 3.87 \\
\hline $\mathrm{CL} / \mathrm{F}\left(\mathrm{L} \mathrm{h}^{-1}\right)$ & 25.4 & $3.6 \%$ & 25.4 & 23.7 to 27.4 \\
\hline $\mathrm{V}_{\mathrm{c}} / \mathrm{F}(\mathrm{L})$ & 2390. & $12.6 \%$ & 2377.8 & 1479 to 3690 \\
\hline $\mathrm{Q} / \mathrm{F}\left(\mathrm{L} \mathrm{h}^{-1}\right)$ & 8.41 & $13.3 \%$ & 9.80 & 6.54 to 16.20 \\
\hline $\mathrm{V}_{\mathrm{p}} / \mathrm{F}(\mathrm{L})$ & 168. & $11.7 \%$ & 175.9 & 135 to 239 \\
\hline ALAG1 & 0.877 & $4.7 \%$ & 0.800 & 0.672 to 0.928 \\
\hline DRUGO ON Ka & 1.89 & $22.0 \%$ & 2.00 & 1.23 to 2.88 \\
\hline DRUGO ON $V_{c} / F$ & 0.216 & $13.1 \%$ & 0.220 & 0.138 to 0.359 \\
\hline DRUG1 ON V $/ F$ & 0.207 & $12.8 \%$ & 0.210 & 0.128 to 0.328 \\
\hline DRUGO ON CL/F & 0.610 & $6.6 \%$ & 0.610 & 0.537 to 0.696 \\
\hline DRUG1 ON CL/F & 0.610 & $5.9 \%$ & 0.610 & 0.545 to 0.695 \\
\hline WT ON V & 0.579 & $25.7 \%$ & 0.590 & 0.191 to 1.06 \\
\hline IIV-Ka & $88.2 \%$ & $24.0 \%$ & $73.1 \%$ & $37.7 \%$ to $115 \%$ \\
\hline IIV-CL/F & $49.1 \%$ & $7.4 \%$ & $46.7 \%$ & $39.8 \%$ to $53.4 \%$ \\
\hline$\omega \mathrm{COV}_{\mathrm{CLF}-\mathrm{V}_{\mathrm{C} / \mathrm{F}}}$ & 0.174 & - & 0.174 & 0.0832 to 0.259 \\
\hline $\mathrm{IIV}-\mathrm{V}_{\mathrm{c}} / \mathrm{F}$ & $40.8 \%$ & $16.1 \%$ & $39.7 \%$ & $22.9 \%$ to $51.4 \%$ \\
\hline IIV-Q/F & 0 FIXED & - & - & - \\
\hline IIV $-V_{p} / F$ & $52.4 \%$ & $29.3 \%$ & $50.1 \%$ & $11.5 \%$ to $90.1 \%$ \\
\hline Prop-Error & 0.216 & $5.0 \%$ & 0.200 & 0.189 to 0.232 \\
\hline Add-Error & 0.303 & $14.5 \%$ & 0.300 & 0.215 to 0.382 \\
\hline
\end{tabular}

${ }^{*}$ Relative SE.

ALAG1, the time lag for absorption; CL/F, apparent clearance of the central compartment; Ka, absorption rate constant; $Q$, transport rate between the central and peripheral compartments; $V_{c} / F$, apparent volume of the central compartment; $V_{p} / F$, apparent volume of the peripheral compartment. 
A

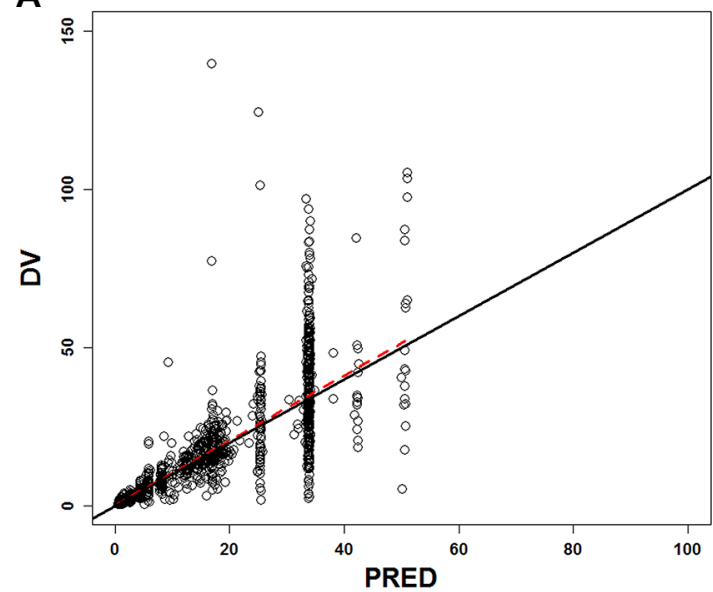

C

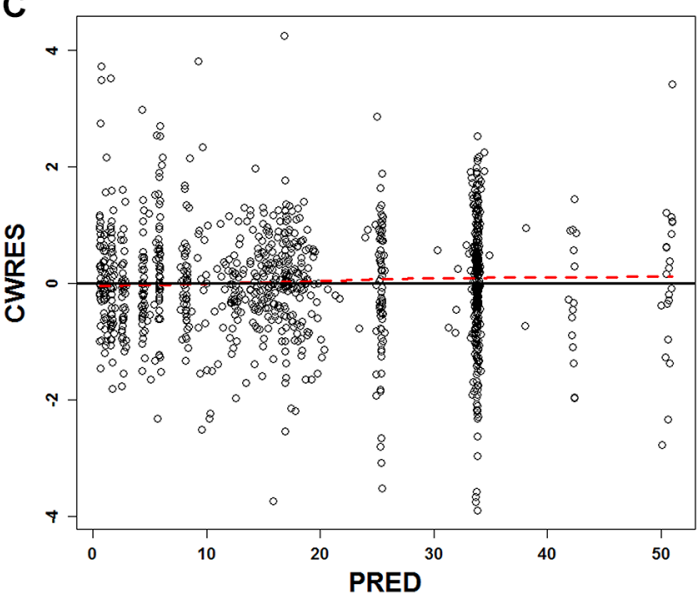

B

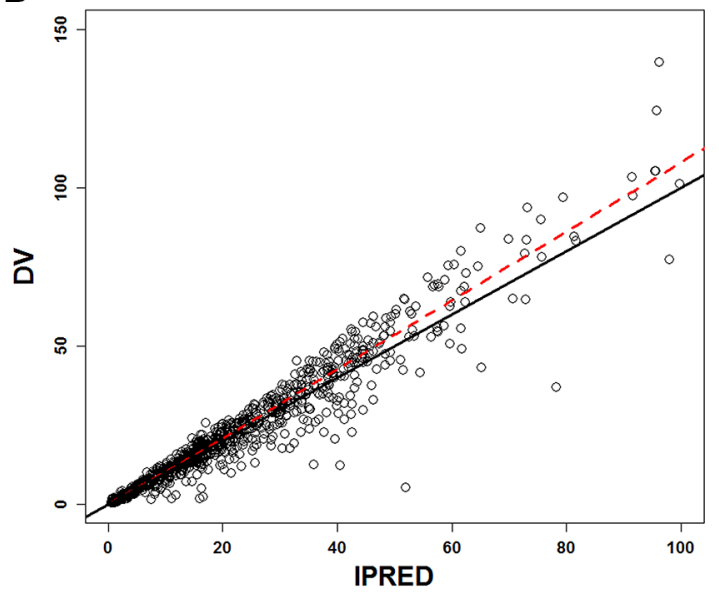

D

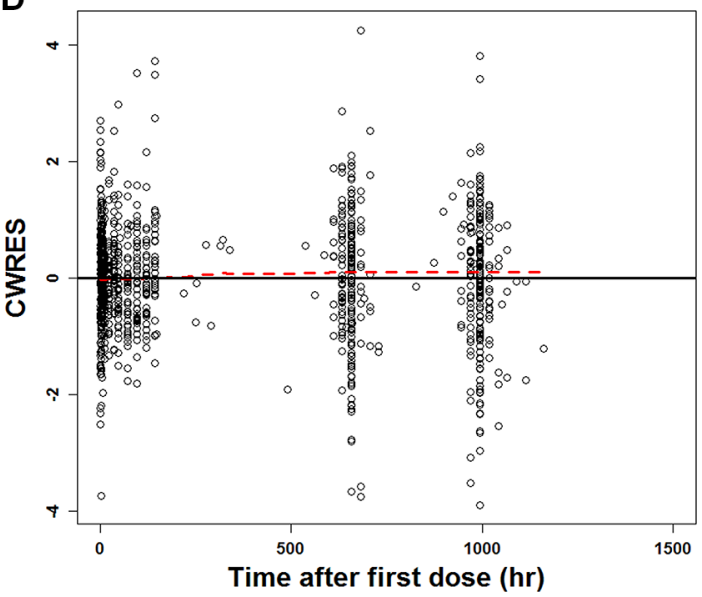

Figure 1 Goodness-of-fit plot for the final model. (A) The observed concentrations (OBS) versus PRED, (B) the OBS versus the Bayesian IPRED, (C) the CWRES versus PRED, (D) the CWRES versus time after the first dose. Solid lines in A and B represent lines of identity. Dotted lines represent Locally estimated scatter plot smoothing (LOESS) smoothing. CWRES, conditional weighted residuals; DV, observed concentrations; IPRED, individual predicted concentrations; PRED, population predicted concentrations.

the final model, suggesting that the model was relatively stable and that most of the parameter results (eg, CL/F, $\mathrm{Vc} / \mathrm{F}, \mathrm{Q} / \mathrm{F}$ and $\mathrm{V}_{\mathrm{p}} / \mathrm{F}$ ) were reliable (table 2). Based on the NPDE results, the points in the QQ graph were mostly distributed around the $\mathrm{Y}=\mathrm{X}$ line (figure 2). In addition, the average value on the bar distribution graph was close to 0 , with a SD near 1 , displaying near-normal distribution. The predicted correlated visual predictive check (VPC) plot for the final model is shown in figure 3 . These results suggest that the model describes the dataset accurately.

\section{DISCUSSION}

A limitation with traditional PK studies is the requirement for rich data sampling. PopPK can be used to analyse both sparse and rich data simultaneously giving a distinct advantage over traditional PK methods. This is useful for infrequent studies for which rich data are difficult to obtain. In addition, PopPK allows the ability to combine data from different centres, groups or batches.

In the current study, we developed a PopPK model for the administration of olanzapine in the Han Chinese population. Both healthy and schizophrenic individuals were included in this study. A two-compartment model with first-order absorption and elimination provided a good description of the PK characteristics of olanzapine. The results suggest that the absorption of olanzapine had a time delay of 0.877 hour after oral administration.

Olanzapine from three different manufacturers was included in this study, and all formulations were oral tablets. Olanzapine was given to healthy subjects in two formulations (\#0 and \#1), while the TDM patients received formulation \#2. When analysing the covariates, it was difficult to separate the influence of subjects and formulation \#2. For formulations \#0 and \#1, which had rich data in the absorption phase, we observed a considerable difference in effect on the absorption rate. The absorption rate of formulation \#1 was approximately twice as high as that of formulation \#0. The clinical treatment monitoring data included only a few sampling points taken during the absorption phase, and most of the samples were collected during the elimination phase. Unfortunately, this data set was not comprehensive 

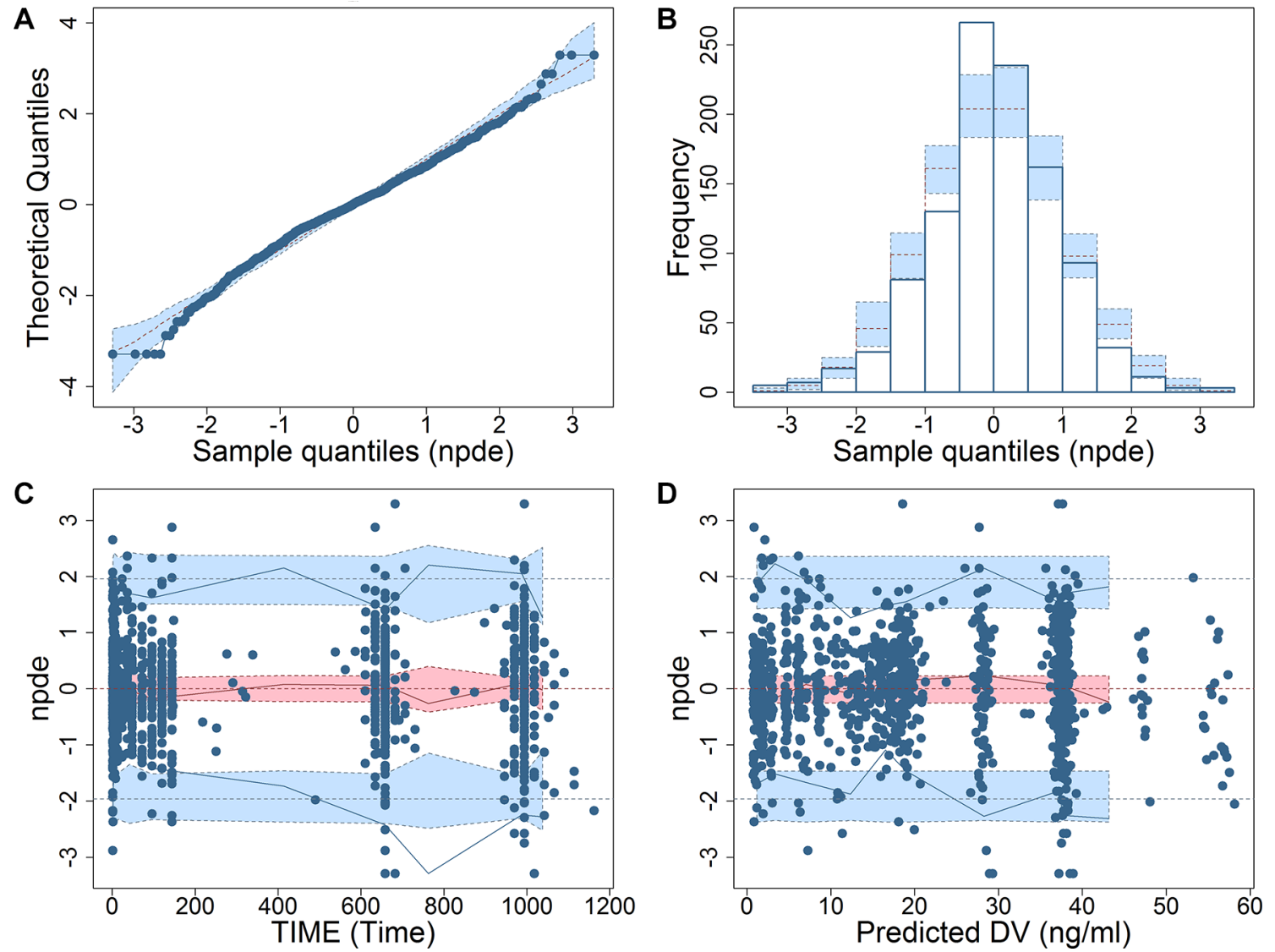

Figure 2 NPDE of the final PopPK model. (A) Q-Q plot of the NPDE, (B) histogram of the NPDE, (C) NPDE versus time after the first dose, (D) NPDE versus PRED. NPDE, normalised prediction distribution errors; PopPK, population pharmacokinetics; PRED, population predicted concentration.

enough to estimate the Ka. Since the data for formulation \#2 were not all trough data, we tried assuming that the Ka of formulation \#2 was the same as formulation \#0 or \#1. The OFV of this model (when assuming that the Ka of formulation \#2 was different from formulation \#1) was higher than that of the assumption (when assuming that the Ka of formulation \#2 was the same as that of formulation \#1), OFV: 4126.82 vs 4133.16 . Therefore, we finally chose the former model.

In PK theory, the parameters of CL and $\mathrm{V}$ are assumed to be independent. However, a correlation between CL and $\mathrm{V}$ is always detected after oral administration. There are several possible reasons for this phenomenon. One is that
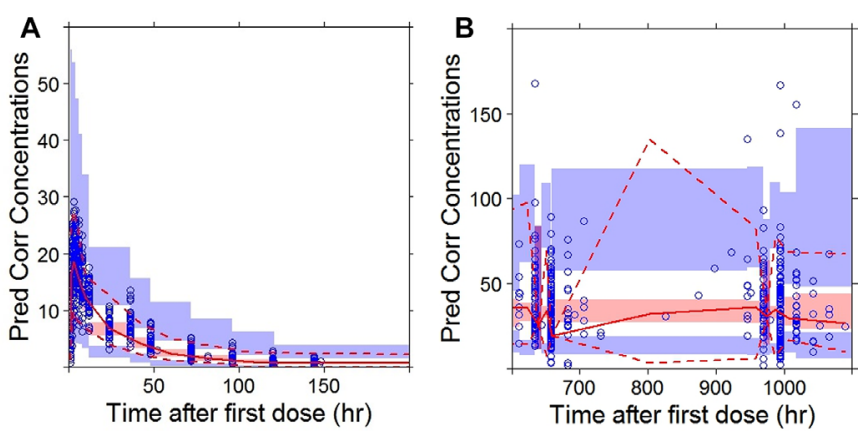

Figure 3 Predicted correlation VPC plot for the final model. (A) Time range: 0-200 hours, (B) time range: 600-1100 hours. the data collected for the oral administration route are not sufficient to support the exact estimation of CL, V and bioavailability $(\mathrm{F})$. Hence, during the modelling process, these three parameters were represented as two parameters, CL/F and Vc/F, both related to bioavailability F. Another reason is that there may be covariates that affect both $\mathrm{CL} / \mathrm{F}$ and $\mathrm{V} / \mathrm{F}$ but are not included in the analysed database or could not be identified because of the limitations of the data. One way to account for this would be to estimate the correlation via the \$OMEGA BLOCK syntax, as presented in this study. As the samplings from healthy subjects were rich and provided a precise estimation of PK parameters, we analysed the data from both healthy subjects and patients with schizophrenia simultaneously. As table 1 shows, the influences of formulation and population on PK parameters were mixed and could not be distinguished due to data limitations. Although we did not identify the exact covariate in this study, these results could provide some suggestions for future studies. Additionally, we performed simulations to identify the influence of absorption rate $(\mathrm{Ka})$ on the PK profile, and the results suggested that the influence of Ka was mainly on $\mathrm{T}_{\max }$ and $\mathrm{C}_{\max }$.

The data from cohort A included only 22 healthy subjects, which was a small sample size and could not realistically reflect the distribution of parameters in clinical 
practice. Consequently, this was the reason we added the data from cohort B to the analysis. In cohort B, drug administration and blood collection times were not as strictly recorded as in cohort $\mathrm{A}$. Therefore, the differences in $\mathrm{V}_{\mathrm{C}} / \mathrm{F}$ and $\mathrm{CL} / \mathrm{F}$ observed in this study may be the results of a combination of formulation, patient population and experimental errors.

A previous PopPK study suggested that gender may affect the elimination rate of olanzapine, as male patients had an elimination rate $30 \%-38 \%$ higher than that of female patients. ${ }^{58}$ However, in our study, we observed no significant gender effect on the olanzapine elimination rate, which was inconsistent with these previous studies. Although the gender ratio in cohort B was almost equal, the subjects in cohort A were all males. This arrangement resulted in asymmetric PK data between the two genders and may have influenced the final results. In addition, the subjects in this study were all Han Chinese, whereas the other two studies involved mostly Caucasian subjects. The racial difference between these two studies may also be responsible for this difference.

Subjects with severe liver function abnormalities were excluded from the study. However, some of the subjects still showed significantly elevated AST and ALT levels. This study revealed that the detected range of transaminase values did not significantly affect the PK parameters. There were no signs of obvious abnormal kidney function in any of the test subjects, and the blood urea nitrogen levels did not affect the PK parameters as long as they remained within normal range. The conclusions were consistent with those of previous studies. ${ }^{15} 16$

Compared with previous studies, this study included a large amount of rich data that provided sufficient information for absorption and distribution phases. We found that using first-order absorption with time delay along with a two-compartment model provided a good fit for modelling the PK characteristics of olanzapine. In previous studies, body weight, gender, age and smoking affected the elimination rate of olanzapine. However, we found that body weight was the only variable that affected the distribution volume of the centre compartment. A PopPK study revealed typical values, with a V/F of $2150 \mathrm{~L}$ and a CL/F of $16.1 \mathrm{~L}$. The $\mathrm{V} / \mathrm{F}$ value of this study was similar to the results of our study when formulation \#2 was used, and the results for CL/F were similar to the results of our study when formulation \#0 and formulation \#1 were used. Another PopPK study of olanzapine produced results similar to ours when formulations \#0 and \#1 were used..$^{5}$ These findings show that our results are consistent with these previous studies. The phenomenon we observed in this study, in which formulations \#0 and \#1 had different $\mathrm{CL} / \mathrm{F}$ and $\mathrm{V}_{\mathrm{C}} / \mathrm{F}$, must be validated in future studies and could potentially be a significant finding. We collected drug effect indicators for olanzapine and established an integral PK-PD model for the Chinese population. We then used the Bayesian method to forecast individual parameters, thus allowing us to choose the best dose regimen for each patient.
This study had certain limitations. For example, smoking has been suggested to greatly affect the metabolism rate of olanzapine ${ }^{517} 18$; however, nearly all the subjects refrained from smoking during the study, and we were not able to collect smoking-related information. The genetic predispositions of the subjects may also affect the metabolism of olanzapine; however, this study did not collect genetic information. In addition, the effects on the $\mathrm{CL} / \mathrm{F}$ and $\mathrm{V}_{\mathrm{C}} / \mathrm{F}$ caused by experimental errors and errors caused by drug type could not be differentiated. The ultimate goal of this analysis was to establish a model to contribute to the individualised dosing of olanzapine in clinical practice. Thus, we focused on the population of patients with schizophrenia. However, as the information in the patient database was quite limited, we had to analyse data from both healthy subjects and patients with schizophrenia simultaneously. The influence of formulation and population on PK parameters was tested. However, the influence was not distinguished due to data limitations. Taken together, these confounding factors require further studies for clarification.

\section{CONCLUSION}

This study used the TDM data of patients with schizophrenia combined with extensive concentration data from healthy volunteers to develop a PopPK model of olanzapine. The results suggest that there may be a delay in absorption after the oral administration of olanzapine. The Ka estimates for formulation \#0 and formulation \#1 were different in this study, and body weight exhibited a considerable impact effect on VC/F. The impact of subjects and formulations requires further study. The PopPK model established in this study likely provides some information for the individualised therapy of olanzapine.

\section{Author affiliations}

${ }^{1}$ The National Clinical Research Center for Mental Disorders \& Beijing Key Laboratory of Mental Disorders, Beijing Institute for Brain Disorders Center of Schizophrenia, Beijing Anding Hospital, Capital Medical University, Beijing, China ${ }^{2}$ Center for Drug Evaluation, China Food and Drug Administration, Beijing, China ${ }^{3}$ Institute of Mental Health, the Sixth Hospital, Key Laboratory of Mental Health, Ministry of Health, Peking University, Beijing, China

${ }^{4}$ School of Pharmaceutical Sciences, Peking University, Beijing, China

${ }^{5}$ Peking-Tsinghua Centre for Life Sciences/ PKU-IDG/McGovern Institute for Brain Research, Peking University, Beijing, China

Acknowledgements We thank Larry House (University of Chicago Biological Sciences) and American Journal Experts for improving the quality of the language.

Contributors AL: Authored the paper, performed the major clinical research work at a subcentre (Beijing Anding Hospital) for cohort B and performed the population pharmacology calculation work. SJ: Performed the major calculation work for population pharmacology and produced the charts and forms. WY: Conducted the clinical research work for cohort B at a subcentre (Peking University Sixth Hospital) and was the director of this subcentre. HY: Performed a major portion of the clinical research work for cohort B at a subcentre (Peking University Sixth Hospital). FD: Implemented the clinical research work for cohort $B$ at a subcentre (Beijing Anding hospital) and conducted some research work for cohort A. CR: Determined the olanzapine concentration detection for cohort B. WLi: Performed major research work for cohort A. DZ: Devised the study for cohort B and was in charge of this cohort. WLu: Created the calculation methods used in this research. CW: Designed 
and was in charge of cohort A. He was also in charge of the subcentre (Beijing Anding Hospital) for cohort B. All authors have read and approved the final manuscript. Larry House (University of Chicago Biological Sciences) and American Journal Experts edited the paper and helped us to improve the quality of the language.

Funding This work was supported by the Beijing Municipal Administration of Hospitals Clinical Medicine Development of Special Funding, grant number ZY201403; the Capital Medical Research Development Fund of China, grant number 2014-2-2122; the National Key Project, grant number 2009AA022702 and the Beijing Science and Technology Plan Project, grant number Z151100004015180. Competing interests None declared.

Patient consent Obtained.

Ethics approval Beijing Anding Hospital and the Sixth Hospital.

Provenance and peer review Not commissioned; externally peer reviewed. Data sharing statement No additional data are available.

Open access This is an open access article distributed in accordance with the Creative Commons Attribution Non Commercial (CC BY-NC 4.0) license, which permits others to distribute, remix, adapt, build upon this work non-commercially, and license their derivative works on different terms, provided the original work is properly cited, appropriate credit is given, any changes made indicated, and the use is non-commercial. See: http://creativecommons.org/licenses/by-nc/4.0/.

\section{REFERENCES}

1. Freedman R. Schizophrenia. N Engl J Med 2003;349:1738-49.

2. Xiang YT, Kreyenbuhl J, Dickerson FB, et al. Use of first- and second-generation antipsychotic medications in older patients with schizophrenia in Asia (2001-2009). Aust N Z J Psychiatry 2012;46:1159-64.

3. Xue HB, Liu L, Zhang H, et al. Olanzapine in Chinese patients with schizophrenia or bipolar disorder: a systematic literature review. Neuropsychiatr Dis Treat 2014;10:841-64.

4. Lieberman JA, Stroup TS, McEvoy JP, et al. Clinical Antipsychotic Trials of Intervention Effectiveness (CATIE) Investigators. Effectiveness of antipsychotic drugs in patients with chronic schizophrenia. N Engl J Med 2005;353:1209-23.
5. Bigos KL, Pollock BG, Coley KC, et al. Sex, race, and smoking impact olanzapine exposure. J Clin Pharmacol 2008;48:157-65.

6. Bergstrom RF CJ, CErimele BJ. Biopharmaceutics integrated: summary volume. Indiana, USA: Lilly Laboratory for Clinical Research: Eli Lilly and Co.

7. Dokoumetzidis A, Aarons L. Propagation of population PK and PD information using a Bayesian approach: dealing with nonexchangeability. J Pharmacokinet Pharmacodyn 2006;33:29-48.

8. Lobo ED, Robertson-Plouch C, Quinlan T, et al. Oral olanzapine disposition in adolescents with schizophrenia or bipolar I disorder: a population pharmacokinetic model. Paediatr Drugs 2010;12:201-11.

9. Yue WH, Wang HF, Sun LD, et al. Genome-wide association study identifies a susceptibility locus for schizophrenia in Han Chinese at 11p11.2. Nat Genet 2011;43:1228-31.

10. Sheiner LB, Beal SL. Evaluation of methods for estimating population pharmacokinetics parameters. I. Michaelis-Menten model: routine clinical pharmacokinetic data. J Pharmacokinet Biopharm 1980;8:553-71.

11. Sheiner LB, Beal SL. Evaluation of methods for estimating population pharmacokinetic parameters. II. Biexponential model and experimental pharmacokinetic data. J Pharmacokinet Biopharm 1981;9:635-51.

12. Sheiner LB, Beal SL. Evaluation of methods for estimating population pharmacokinetic parameters. III. Monoexponential model: routine clinical pharmacokinetic data. J Pharmacokinet Biopharm 1983;11:303-19.

13. Ette El. Stability and performance of a population pharmacokinetic model. J Clin Pharmacol 1997;37:486-95.

14. Comets E, Brendel K, Mentré F. Computing normalised prediction distribution errors to evaluate nonlinear mixed-effect models: the npde add-on package for R. Comput Methods Programs Biomed 2008;90:154-66.

15. Thomasson HR CJ, Bergstrom RF. Oral olanzapine: safety and pharmacokineticstudy in subjects with cirrhosis. Indiana, USA: Eli Lilly and Co: Lilly Laboratory for Clinical Research, 1996.

16. Bergstrom RF CB. Olazapine in subjects with and without renal failure. Indiana, USA: Eli Lilly and Co: Lilly Laboratory for Clinical Research, 1996.

17. Callaghan JT, Bergstrom RF, Ptak LR, et al. Olanzapine. Pharmacokinetic and pharmacodynamic profile. Clin Pharmacokinet 1999;37:177-93.

18. Lucas RA, Gilfillan DJ, Bergstrom RF. A pharmacokinetic interaction between carbamazepine and olanzapine: observations on possible mechanism. Eur J Clin Pharmacol 1998;54:639-43. 